\title{
Aortic valve-in-valve replacement
}

Surgery for aortic valve disease commonly involves the implantation of a bioprosthetic valve, but these devices have limited durability and are expected to degenerate and fail within $10-20$ years, which necessitates reoperation in these patients. However, registry data analyzed by Dr Danny Dvir and colleagues indicate that "the 'off-label' use of transcatheter aortic valve implantation (TAVI) within failed surgically-inserted bioprosthetic valves ('valve-in-valve') is technically feasible", and might obviate the need for reoperation.

The Global Valve-in-Valve Registry was initiated in December 2010 and, at the time of the analysis, included 202 patients with bioprosthetic valves that had deteriorated (mean age $77.7 \pm 10.4$ years, $52.5 \%$ men) from 38 centers in Australia, Europe, the Middle East, New Zealand,

Original article Dvir, D. et al. Transcatheter aortic valve replacement for degenerative bioprosthetic surgical valves: results from the Global Valve-in-Valve Registry. Circulation doi:10.1161/CIRCULATIONAHA.112.104505 and North America. The median time from the previous aortic valve surgery to the valve-in-valve procedure was 9 years. Valve failure was classified as stenosis (42.1\%), regurgitation (33.7\%), or a combination of both (24.3\%).

Overall, 124 and 78 patients had a new CoreValve $^{\circledast}$ (Medtronic, Minneapolis, MN, USA) or Edwards SAPIEN ${ }^{\circledR}$ valve (Edwards Lifesciences, Irvine, CA, USA) implanted, respectively, with a combined 93.1\% procedural success rate. Common complications included initial device malposition (15.3\%) and coronary ostial obstruction (3.5\%). Furthermore, a high rate of elevated postprocedural aortic gradients was observed. At 30-day follow-up, all-cause mortality was $8.4 \%$, and $83.7 \%$ of patients were in NYHA class I or II. At 1-year follow-up (available for 87 patients), survival was $85.8 \%$, with no significant difference between the CoreValve ${ }^{\varpi}$ and Edwards SAPIEN ${ }^{\circledR}$ devices.

The investigators caution that "there is not enough data to justify valve-in-valve

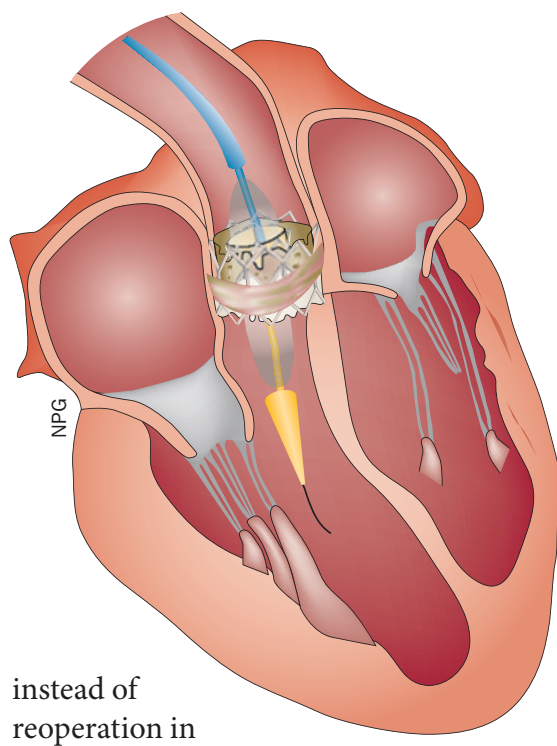

most high-risk patients

with failed aortic bioprostheses.

Nevertheless, valve-in-valve could be an acceptable approach in carefully selected high-risk patients." According to Dr Dvir, the registry "includes by now almost 500 aortic cases and almost 100 mitral valvein-valve procedures... Our next step is to evaluate long-term results."

Gregory B. Lim 